\title{
Pontoscolex corethrurus (Annelida: Oligochaeta) indicador de la calidad del suelo en sitios de Eucalyptus grandis (Myrtacea) con manejo tumba y quema
}

\author{
Sheila Uribe ${ }^{1,2}$, Esperanza Huerta ${ }^{1}$, Violette Geissen ${ }^{1,3}$, Manuel Mendoza $^{4}$, Roberto Godoy ${ }^{5}$ \& \\ Aarón Jarquín ${ }^{1}$ \\ 1. Departamento de Agroecología, El Colegio de Frontera Sur, unidad Villahermosa. Carr. Villahermosa-Reforma km. \\ 15.5. Ra. Guineo 2da. Secc.86280. Centro, Tabasco, México; sheirylopez@gmail.com, ehuerta@ecosur.mx, \\ ajarquin@ecosur.mx \\ 2. Universidad Politécnica del Centro, Carr. Federal Villahermosa-Teapa km. 22.5. Tumbulushal, Centro, Tabasco, \\ México. 86290. \\ 3. Wageningen University, LAD group,6700AA Wageningen, The Netherlands; vgeissen@web.de \\ 4. Departamento de Aprovechamiento de Recursos Acuáticos, El Colegio de Frontera Sur, unidad Villahermosa. Carr. \\ Villahermosa-Reforma km. 15.5. Ra. Guineo 2da. Secc. 86280. Centro, Tabasco, México; mcarranza@ecosur.mx \\ 5. Instituto de Botánica, Facultad de Ciencias. Universidad Austral de Chile, Casilla 567-Valdivia; rgodoy@uach.cl
}

$$
\text { Recibido 03-X-2011. Corregido 08-V-2012. Aceptado 07-VI-2012. }
$$

\begin{abstract}
Pontoscolex corethrurus (Annelidae: Oligochaeta) soil quality indicator in Eucalyptus grandis (Myrtacea) sites with slash and burn management. Soil burning has been used in agricultural and forestry systems as a fundamental technique to clean the land and add some nutrients to the soil. In addition, earthworms are known to promote various soil functions since they contribute to aeration and organic matter and nutrients availability to other soil organisms. This study evaluated the effects of tropical forest crops management with presence-absence of Eucalyptus grandis on earthworm population in Huimanquillo, Tabasco, Mexico. Three sites (average area of 1-1.5ha each) with different management conditions were considered for soil and earthworm sampling (two depths and six replicates): without vegetation (SV) and recent slash-burned (38 days), forest crops of five years of production of E. grandis (Euc), and secondary vegetation of 15 years (Acah). Soil physico-chemical properties (apparent density, humidity, texture, $\mathrm{pH}$, Ntot, OM, P, K, cationic capacity) were also evaluated, and earthworms were collected at the end of the rainy season (august-october 2007). We found that the sites soil is an acrisol acid, with $\mathrm{pH} 3.0-4.5$ in the first $30 \mathrm{~cm}$ depth. Organic matter content (OM) and total nitrogen (Ntot) in the recently burned sites were significantly lower (6-8\% y $0.19-0.22 \%$, respectively) than in sites with vegetation (OM=9-11\%; el Ntot $=0.27-0.33 \%)$. Only one species $(P$. corethrurus $)$ was found in all the sampled areas, where most of the individuals were at juvenile stage $(80 \%)$. The highest densities and biomass were found in Euc. treatment $\left(166.4 \mathrm{ind} / \mathrm{m}^{2}\right.$ y $\left.36.8 \mathrm{~g} / \mathrm{m}^{2}\right)$ followed by Acah $\left(138.7 \mathrm{ind} / \mathrm{m}^{2}\right.$ y $19.1 \mathrm{~g} / \mathrm{m}^{2}$ respectively), while the SV treatment showed of about an $80 \%$ reduced earthworm populations when compared to other treatments. Even though 15 years have passed over the secondary vegetation (Acah) still some perturbations were observed as the low abundance of the oligochaeta group. We concluded that the management used to culture $E$. grandis produces negative effects over the abundance and diversity of earthworms and soil nutrient availability. Rev. Biol. Trop. 60 (4): 1543-1552. Epub 2012 December 01.
\end{abstract}

Key words: Pontoscolex corethrurus, earthworms, deforestation and slash burning, Eucalyptus grandis, management, soil fertility.

Desde tiempos prehispánicos en México y Centroamérica se ha utilizado el fuego en los sistemas agrícolas y forestales como una técnica fundamental para limpiar los terrenos e incorporar algunos nutrientes al suelo (FAO 2009). La materia orgánica calcinada facilita la disponibilidad de fósforo, potasio, calcio y magnesio, acelera el proceso de remoción 
y descomposición de los residuos vegetales por el incremento de la actividad microbiana (Wojtkowski 2002, Wilson et al. 2002). Por tanto, la biomasa microbiana se caracteriza por una estrecha relación $\mathrm{C} / \mathrm{N}$, al metabolizar parcialmente compuestos orgánicos (Mengel, 1996). Sin embargo, su empleo es cuestionado en el ámbito ambiental ya que el manejo intensivo de este disminuye o anula los tiempos de reposo de los ecosistemas, lo cual incrementa la degradación de los suelos, altera la aireación, la infiltración, la densidad de estos y la textura al aumentar los contenidos de limo y arena, mientras se reducen los contenidos de arcilla en la superficie (Román-Cuesta et al. 2000, PonceMendoza et al. 2006). Además, un mal manejo del fuego puede provocar erosión, lixiviación de nutrientes y elevar la temperatura del suelo causada por la radiación solar post-quema (Lal 1987). Desde el punto de vista socioeconómico el eucalipto, en específico E. grandis ha demostrado ser un cultivo netamente industrial, sin proporcionar satisfactores o beneficios de una planta multipropósito a nivel local (Kanowski \& Savill 1990); su cultivo requiere de grandes inversiones (Turnbull 1990).

En Tabasco, como en otras partes del sureste mexicano se emplea la técnica de tumba y quema en la mayoría de los sistemas forestales, tal es el caso del municipio de Huimanguillo donde se encuentran establecidos monocultivos de especies forestales maderables como: cedro (Cedrela odorata L.), macuilí (Tabebuia rosea Bertol DC), melina (Gmelina arborea Roxb.) y eucaliptos (Eucalyptus urophylla S.T. Blakede y E. grandis W. Hill ex Maiden) con los cuales se han generado proyectos productivos en el estado y de los que sobresale el cultivo de eucaliptos, que en conjunto fomentan la industria papelera a nivel nacional (Ceccon \& Martínez-Ramos 1999, Martínez-Ruíz et al. 2006, Téllez et al. 2008). Sin embargo, esta planta puede provocar efectos secundarios en las áreas donde se cultiva, tales como: desecación, acidificación de los suelos, producción de efecto alelopático sobre numerosas especies de flora autóctona, formando una vasta cadena de raíces que absorbe grandes cantidades de agua (Ribeiro et al. 2002).

El manejo del uso del fuego en los sistemas forestales y otros sistemas agrícolas varían en intensidad dependiendo de las condiciones ambientales del lugar y del tipo o composición del residuo vegetal a quemar (Gibbons et al. 2000). Una quema controlada de baja intensidad causa menos pérdidas de nutrientes y cambios en las propiedades físicas del suelo en comparación con los incendios de alta intensidad (Rab 1996, Fernández et al. 2004). Por ejemplo, algunos investigadores indican que durante las quemas se incrementa la temperatura del suelo donde se crean condiciones favorables para la actividad microbiana al liberarse amonio y fósforo con mayor facilidad (Wilson et al. 2002, Knoepp et al. 2004). Asimismo, la transformación de la materia orgánica en el suelo provocada por una quema comienza a partir de $\operatorname{los} 210^{\circ} \mathrm{C}$, lo que aumenta la concentración de amonio y disminuye las concentración de nitratos (Gimeno-García et al. 2000). Temperaturas entre $200^{\circ}-300^{\circ} \mathrm{C}$ implica que el $80 \%$ de las sustancias orgánicas sean destruidas por destilación (Knoepp et al. 2004, Yang et al. 2005). Mientras que el nitrógeno del suelo es más estable al calor que el carbono, con pérdidas menores hasta $\operatorname{los} 450^{\circ} \mathrm{C}$ (Villar et al. 2004), y donde la mayor concentración del nitrógeno en el suelo está formando parte de los ácidos húmicos (Almendros et al. 1990, Jaramillo et al. 2003, Korb et al. 2004). El manejo de la quema con fines de preparación del terreno forma del parte de la técnica nómada o transhumante de roza-tumba-quema (GomezPompa 1971), la cual incluye la selección del sitio, el aclareo del bosque mediante el corte de arbustos, y bejucos, el derribo de los árboles y la quema de los residuos secos (Ochoa-Gaona et al. 2007).

Las lombrices de tierra participan y promueven diversas funciones del suelo al modificar su entorno (Lavelle et al. 1997). Estos organismos promueven la aeración e infiltración del suelo con sus galerías e intervienen en la estructura al producir pequeños o grandes agregados, dando lugar a que la materia orgánica se estabilice en sus excretas (turrículos) y 
que se promueva la disponibilidad de materia orgánica y nutrientes para otros organismos del suelo (Jouquet et al. 2006, Edwards \& Bohlen 1996, Brown et al. 1999, Bouché, 1984). La presencia de oligoquetos en los ecosistemas pueden indicar fertilidad del suelo, ya que estos organismos transportan, mezclan y entierran los residuos vegetales de la superficie al interior del suelo (Lavelle \& Spain 2001, Huerta et al. 2007a, Huerta et al. 2007b). Este trabajo tiene como objetivo caracterizar la comunidad de lombrices bajo sitios con diferentes periodos de establecimiento y manejo de plantaciones de Eucalyptus grandis (sin vegetación, con 5 años y 15 años), que han pasado por el proceso de tumba y quema.

\section{MATERIALES Y MÉTODOS}

Área de estudio: Se seleccionaron tres sitios con o sin plantaciones de Eucalyptus grandis con diferentes periodos de permanencia de las plantaciones (7-8 años) y con manejo de tumba y quema. El primer tratamiento es un sitio sin vegetación (SV), con 38 días de haber sido manejado con tumba y quema después de la cosecha de los árboles. El segundo tratamiento es un sitio con una plantación de $E$. grandis (Euc) con más de cinco años de edad. Por último, el tercer tratamiento es un acahual (Acah) con más de 15 años, cuya vegetación surge de un proceso de sucesión ecológica o regeneración natural, en un terreno de uso agrícola-forestal sometido a desmontes y/o quemas, y luego abandonado. Su composición florística es variable al establecerse plantas pioneras y de crecimiento rápido (ciperáceas, compuestas, leguminosas y gramíneas), la especie arbórea dominante fue Curatella americana y un $10 \%$ de árboles de $E$. grandis. Todos los sitios tenían una extensión variable entre 1-1.5ha y se encuentran ubicados dentro de las áreas de la empresa Forestaciones Operativas Mexicanas (FOMEX) en el municipio de Huimanguillo (17 $83^{\prime} 04^{\prime \prime} \mathrm{N}-93^{\circ} 64^{\prime} 88^{\prime \prime}$ W), Tabasco, México.

El tipo de clima es cálido húmedo Am(f) presenta abundantes lluvias en verano con cambios térmicos en diciembre-enero, la temperatura media anual es mayor de $26^{\circ} \mathrm{C}$, siendo la máxima media mensual en Mayo con $29^{\circ} \mathrm{C}$ y la precipitación media anual de 2343.7mm (INEGI 2005). La vegetación que predominaba antes de cultivar eucalipto era de selva mediana sub-perennifolia. Actualmente, solo se pueden encontrar relictos de esta selva sobre los márgenes de los arroyos en el municipio (Palma-López et al. 2007, INEGI 2001).

Recolecta de oligoquetos: La recolecta de oligoquetos se realizó al final de la época de lluvias, entre agosto y octubre 2007. En cada sitio se realizaron tres cuadrantes distribuidos al azar de 50 por $20 \mathrm{~m}^{2}$, dentro de cada cuadrante se hicieron seis monolitos al azar de $25 \times 25 \times 30 \mathrm{~cm}$ de profundidad, para un total de 18 monolitos por tratamiento, cada monolito se dividió en dos estratos: $0-10$ y $10-30 \mathrm{~cm}$ de profundidad de acuerdo al método TSBF (Anderson \& Ingram 1993). La recolecta de lombrices fue manual de acuerdo al método de Satchell (1967). Los oligoquetos fueron colocados en formol (4\%) para su identificación, conteo y obtención de biomasa fresca. Se identificaron a nivel de especies de acuerdo a criterios y claves taxonómicas (Fragoso 1993, Fragoso 2001).

Muestreo del suelo: Se tomaron muestras de suelo de cada uno de los estratos de los monolitos de donde fueron extraídas las lombrices $(0-10 \mathrm{~cm}$ y de $10-30 \mathrm{~cm})$, para determinar los parámetros físico-químicos del suelo de acuerdo a los métodos de análisis de la Sociedad Americana de la Ciencia del Suelo (Sparks 1996) y la categorización de los contenidos con la norma oficial mexicana para suelos no volcánicos NOM-021-RECNAT-2000 (SEMARNAT 2000) densidad aparente (DA; Porta et al. 1999), textura (Bouyoucos 1962), humedad relativa (Sparks 1996), $\mathrm{pH}$ en $\mathrm{H}_{2} \mathrm{O}$ (relación 1:2; Willard et al. 1974), materia orgánica (MO; Walkley \& Black 1934), nitrógeno total (Ntot; SEMARNAT, 2000), fósforo (P; Olsen \& Dean 1965), potasio (K; Bigham \& Bartels 1996) y capacidad de intercambio catiónico (CIC; Bigham \& Bartels 1996). 
Durante el muestreo no se aplicaron fertilizantes. Sin embargo, las áreas que son recién quemadas se dejan reposar durante un mes para posteriormente aplicar fertilizantes al inicio de la plantación, una dosis de 120-180g/planta del fertilizante sólido fosfato diamónico (DAP) 18-46-00 (NPK) y diversos químicos que se utilizan para el control de plagas $(3-7 \mathrm{~L} / \mathrm{ha}$ de Roundup y 2-3kg/ha de Simazina) de 15-30 días antes de la plantación (información proporcionada por los propietarios).

En cada uno de los sitios se calcularon las medias y desviaciones estándar (D.E.) de la biomasa y la densidad de oligoquetos, y de las propiedades físico-químicas del suelo. Asimismo, se analizaron las variables antes mencionadas entre los sitios con pruebas de Kruskall-Wallis (KW) y pruebas a posteriori de Mann-Whitney (U) con $\mathrm{p}<0.05$. Por último, se aplicaron correlaciones de Spearman entre las propiedades físico-químicas del suelo y abundancia de oligoquetos (StatSoft 2006).

\section{RESULTADOS}

La única especie de oligoqueto encontrada en los tres sitios fue Pontoscolex corethrurus con la mayoría de los individuos en etapa juvenil. El sitio Euc presentó la mayor densidad y biomasa de oligoqueta en los dos estratos (superior media $164 \mathrm{ind} / \mathrm{m}^{2}$, dev. estándar $78 \mathrm{ind} / \mathrm{m}^{2}$ y $37 \mathrm{ind} / \mathrm{m}^{2}$, dev. estándar $21 \mathrm{~g} / \mathrm{m}^{2}$ e inferior 51 ; 39ind $/ \mathrm{m}^{2}$ y $13 ; 12 \mathrm{~g} / \mathrm{m}^{2}$ respectivamente). Sin embargo, el sitio SV en el estrato superior presentó significativamente mayor densidad de oligoqueta pero en el estrato inferior el SV no fue significativamente diferente de Euc. La biomasa en el estrato superior fue significativamente menor en SV que en el Euc (Cuadro 1). En caso particular del sitio Acah fue observada una densidad promedio media $\left(139 \mathrm{ind} / \mathrm{m}^{2}\right)$. Sin embargo, este valor tuvo la mayor desviación debido a la distribución agrupada de $P$. corethrurus en este ambiente (Cuadro 1).

El suelo predominante en el área fue del tipo Acrisol, con clase textural franco arenoso en los sitios. Sin embargo, el sitio sin vegetación (SV) presentó menor porcentaje de limo en ambos estratos con respecto a los sitios con vegetación, donde en el estrato superior el $\mathrm{Acah}=21-43 \%$, Euc=26-38\% y SV=19$30 \%\left(\mathrm{U}_{\text {Acah-SV }}=96.50, \mathrm{p}=0.038 ; \mathrm{U}_{\text {Euc-SV }}=56.00\right.$, $\mathrm{p}=0.000)$, en el estrato inferior Acah $=25-43 \%$, $\mathrm{Euc}=29-41 \%$ y $\mathrm{SV}=20-32 \%\left(\mathrm{U}_{\text {Acah-SV }}=68.50\right.$, $\mathrm{p}=0.003 ; \mathrm{U}_{\text {Euc-SV }}=43.00, \mathrm{p}=0.000$ ). El mayor contenido de arena se encontró en el estrato inferior del sitio SV (60.1\%) en comparación con Acah y Euc (51.9\% respectivamente) con diferencias significativas $\left(\mathrm{U}_{\mathrm{Acah}-\mathrm{SV}}=86.00\right.$, $\left.\mathrm{p}=0.015 ; \mathrm{U}_{\text {Euc-SV }}=60.50, \mathrm{p}=0.001\right)$, mientras que en el primer estrato solo se evidenció diferencia entre Euc y SV ( $U=67.50, p=0.003)$. La humedad en el suelo fue homogénea para todos los sitios en un rango entre $30-40 \%$. Sin embargo, D.A. en el estrato superior fue heterogénea entre los tres sitios significativamente

\section{CUADRO 1}

Densidad y biomasa de lombrices de tierra en los sitios de muestreo bajo dos diferentes profundidades del suelo en Huimanguillo, Tabasco

TABLE 1

Density and biomass of earthworms in the sampling sites under two different soil depths in Huimanguillo, Tabasco

\begin{tabular}{|c|c|c|c|c|}
\hline \multirow[t]{2}{*}{ Sitio } & \multicolumn{2}{|c|}{ Densidad (ind $\left./ \mathrm{m}^{2}\right)^{*}$} & \multicolumn{2}{|c|}{ Biomasa fresca $\left(\mathrm{g} / \mathrm{m}^{2}\right)^{*}$} \\
\hline & $0-10 \mathrm{~cm}$ & $10-30 \mathrm{~cm}$ & $0-10 \mathrm{~cm}$ & $10-30 \mathrm{~cm}$ \\
\hline Acah & $139 \pm 148 \mathrm{a}$ & $49 \pm 51 \mathrm{a}$ & $19 \pm 20 \mathrm{a}$ & $9 \pm 9$ \\
\hline Euc & $164 \pm 78 \mathrm{a}$ & $51 \pm 39 a b$ & $37 \pm 21 \mathrm{~b}$ & $13 \pm 12$ \\
\hline SV & $16 \pm 35 b$ & $20 \pm 25 \mathrm{ac}$ & $5 \pm 13 c$ & $7 \pm 2$ \\
\hline
\end{tabular}

*=Promedio \pm desviación estándar.

Los valores entre los sitios seguidos por la misma letra no son significativamente diferentes ( $<<0.05)$. Acah: Acahual, Euc: Plantación de Eucalyptus grandis con 5 años, SV: sitio sin vegetación. 
$\left(\mathrm{U}_{\text {Acah-Euc }}=92.00, \mathrm{p}=0.027 ; \mathrm{U}_{\text {Acah-SV }}=41.00\right.$, $\left.\mathrm{p}=0.000 ; \mathrm{U}_{\text {Euc-SV }}=88.00, \mathrm{p}=0.019\right)$, mientras que en el estrato inferior el tamaño de los agregados fueron significativamente mayores en el sitio SV respecto al Acah ( $\mathrm{U}=64.00$, $\mathrm{p}=0.002$; Cuadro 2).

El pH de los sitios fue fuertemente ácido en los dos estratos del suelo donde la mayor acidez se registró en los sitios con eucalipto respecto al que no tiene $\left(0-10 \mathrm{~cm}\right.$ : $\mathrm{U}_{\text {Acah- }}$ $\mathrm{sV}=50.00, \mathrm{p}=0.000 ; \mathrm{U}_{\text {Euc-SV }}=27.00, \mathrm{p}=0.000$; $10-30 \mathrm{~cm}: \quad \mathrm{U}_{\text {Acah-SV }}=46.00, \mathrm{p}=0.000 ; \mathrm{U}_{\text {Euc- }}$ $\mathrm{sv}=44.50, \mathrm{p}=0.000)$. El contenido de $\mathrm{MO}$ en el área fue significativamente mayor en el sitio Acah en ambos estratos con respecto al sitio SV $(\mathrm{U}=52.00, \mathrm{p}=0.000 ; \mathrm{U}=67.00, \mathrm{p}<0.002$ respectivamente). Se observó que la concentración de Ntot fue significativamente diferente entre los sitios con y sin eucalipto en el estrato superior del suelo $\left(\mathrm{U}_{\text {Acah-Euc }}=99.00, \mathrm{p}=0.046 ; \mathrm{U}_{\text {Acah- }}\right.$ $\left.\mathrm{SV}=16.00, \mathrm{p}=0.000 ; \mathrm{U}_{\text {Euc-SV }}=84.00, \mathrm{p}=0.014\right)$, donde el Acah presentó niveles altos (0.24$42 \%)$, el Euc intermedios (0.19-0.35\%) y SV bajos (0.19-0.23\%, Cuadro 3). En el estrato inferior la concentración de Ntot fue significativamente diferente mayor en Acah con respecto a los sitios Euc $(\mathrm{U}=45.00, \mathrm{p}=0.000)$ y $\mathrm{SV}(\mathrm{U}=18.50, \mathrm{p}=0.000)$. La concentración de potasio fue muy bajo en los tres sitios $(<0.2 \mathrm{cmol} / \mathrm{kg})$, sin embargo, los sitios con vegetación presentaron perdidas significativamente mayores de $\mathrm{K}$ que el sitio $\mathrm{SV}$ en el estrato superior del suelo $\left(\mathrm{U}_{\mathrm{Acah}-\mathrm{SV}}=72.00\right.$, $\mathrm{p}=0.004 ; \quad \mathrm{U}_{\text {Euc-SV }}=18.00, \mathrm{p}=0.000$ ), mientras que en el inferior todos los sitios fueron significativamente diferentes entre sí $\left(\mathrm{U}_{\mathrm{Acah}}\right.$ ${ }_{\text {Euc }}=63.00, p=0.002 ; U_{\text {Acah-SV }}=90.00, p=0.023$; $\mathrm{U}_{\text {Euc-SV }}=13.00, \mathrm{p}=0.000$; Cuadro 3). El contenido de fósforo del suelo en el estrato superior presento un valor intermedio de $5.5-11 \mathrm{mg} \mathrm{kg}^{-1}$, y en inferior hasta $<5.5 \mathrm{mg} / \mathrm{kg}$. Por último, el contenido bajo de CIC de $5-15 \mathrm{cmol} / \mathrm{kg}$ en el estrato inferior del área indica que el tipo de arcilla presente es "caolinita", el Acah presentó mayor CIC que en el Euc (U=86.00, $\mathrm{p}=0.016 \mathrm{y}$ el SV (U=90.00, $\mathrm{p}=0.023$; Cuadro 3).

La densidad de oligoquetos en el estrato superior del suelo se correlacionó inversamente con el $\mathrm{pH}$ y el $\mathrm{K}$ del suelo $\left(\mathrm{r}^{2}\right.$ de Spearman $=-0.40$ y -0.43 respectivamente, $p<0.05)$, y positivamente con el Ntot y el limo del suelo $\left(\mathrm{r}^{2}\right.$ de Spearman $=0.29$ y 0.28 respectivamente, $\mathrm{p}<0.05$ ). Asimismo, la densidad de oligoquetos en el estrato inferior del suelo se relacionó inversamente con el $\mathrm{K}$ del suelo $\left(\mathrm{r}^{2}\right.$ de Spear$\operatorname{man}=-0.34, \mathrm{p}<0.05$ ). Por último como era de esperarse la biomasa fresca de oligoquetos en el estrato superior del suelo se relacionó inversamente con el $\mathrm{pH}$ y el $\mathrm{K}\left(\mathrm{r}^{2}\right.$ de Spearman $=-0.38, \mathrm{p}<0.05)$.

\section{DISCUSIÓN}

Los Acrisoles son suelos degradados, lixiviados, ácidos y pobres en nutrientes (Aguilera 1989), se caracterizan por presentar una

CUADRO 2

Características físicas de suelos en tres sitios a dos profundidades de muestreo del suelo, en Huimanguillo, Tabasco

TABLE 2

Physical characteristics of soil in three sampling sites at two depths of soil sampling in Huimanguillo, Tabasco

\begin{tabular}{lcccccccccc} 
& \multicolumn{2}{c}{ Humedad $(\%)^{*}$} & \multicolumn{2}{c}{ D.A. $\left(\mathrm{g} / \mathrm{cm}^{3}\right)^{*}$} & \multicolumn{2}{c}{ Arcilla $(\%)^{*}$} & \multicolumn{2}{c}{ Limo $(\%)^{*}$} & \multicolumn{2}{c}{ Arena $(\%)^{*}$} \\
Sitio & $0-10 \mathrm{~cm}$ & $10-30 \mathrm{~cm}$ & $0-10 \mathrm{~cm}$ & $10-30 \mathrm{~cm}$ & $0-10 \mathrm{~cm}$ & $10-30 \mathrm{~cm}$ & $0-10 \mathrm{~cm}$ & $10-30 \mathrm{~cm}$ & $0-10 \mathrm{~cm}$ & $10-30 \mathrm{~cm}$ \\
Acah & $33.5 \pm 5.1$ & $36.0 \pm 3.9$ & $0.84 \pm 0.2 \mathrm{a}$ & $0.93 \pm 0.1 \mathrm{a}$ & $11.9 \pm 4$ & $14.1 \pm 4$ & $30.0 \pm 9 \mathrm{a}$ & $34.0 \pm 9 \mathrm{a}$ & $58.1 \pm 11 \mathrm{a}$ & $51.9 \pm 11 \mathrm{a}$ \\
Euc & $31.1 \pm 4.4$ & $37.6 \pm 4.7$ & $0.95 \pm 0.1 \mathrm{~b}$ & $1.01 \pm 0.1 \mathrm{ab}$ & $12.2 \pm 3$ & $13.4 \pm 3$ & $32.2 \pm 6 \mathrm{a}$ & $34.8 \pm 6 \mathrm{a}$ & $55.6 \pm 7 \mathrm{~b}$ & $51.9 \pm 8 \mathrm{a}$ \\
SV & $33.4 \pm 8.0$ & $35.7 \pm 3.7$ & $1.04 \pm 0.1 \mathrm{c}$ & $1.07 \pm 0.1 \mathrm{~b}$ & $12.5 \pm 4$ & $14.0 \pm 3$ & $24.6 \pm 5 \mathrm{~b}$ & $25.9 \pm 6 \mathrm{~b}$ & $62.9 \pm 6 \mathrm{c}$ & $60.1 \pm 5 \mathrm{~b}$ \\
\hline
\end{tabular}

*=Promedio \pm desviación estándar.

a, b y c=Pruebas de Mann-Whitney, donde letras iguales no diferencia significativa entre los sitios, $\mathrm{p}<0.05$. Acah: Acahual, Euc: Plantación de Eucalyptus grandis con 5 años, SV: sitio sin vegetación. 
coloración superficial oscura sobre un basamento de color amarillo o rojo, material geológico del Pleistoceno y Terciario, característico en la zona de planicie y algunos lomeríos de Huimanguillo (Palma-López \& Cisneros 2000). Son de reacción ácida lo cual es indicador de fertilidad baja, tienen problemas de manejo por la toxicidad de hierro, aluminio y fijación de fósforo, los cuales provocan restricciones en su uso agrícola (INEGI 2005). Marín (2006) en un estudio de caracterización, clasificación y cartografía de suelos citrícolas en la sabana de Huimanguillo, encontró suelos con un $\mathrm{pH}$ entre 4.5 y 6.0 a $40 \mathrm{~cm}$ de profundidad del suelo. Este resultado es considerado una mínima variación de lo encontrado en nuestro estudio en los sitios con vegetación de eucalipto (Euc y Acah=pH 3.7) y los de reciente quema con un aumento mínimo de $\mathrm{pH} 4.4$ del suelo. Este patrón puede deberse al incinerarse la hojarasca el $\mathrm{N}$ y los componentes de ácidos orgánicos, regresan en forma de cationes inorgánicos al suelo $\left(\mathrm{K}^{+}\right.$y $\left.\mathrm{Ca}^{2+}\right)$, los cuales producen un efecto alcalinizante (Gleissman 2002, Jaramillo et al. 2003, Korb et al. 2004). Sin embargo, con el paso del tiempo cuando esta se reincorpora en forma de cenizas en el sistema los factores físico-químicos, así como el manejo del nuevo cultivo presentará otra vez cierta tendencia de acidificación de suelos (Certini 2005, Nadel et al. 2007).

Con respecto a los contenidos de nutrientes en el suelo, la materia orgánica y el nitrógeno disminuyeron en los sitios recientemente quemados, pero una vez que se establecen las plantaciones tienden a incrementar sus niveles, tal es el caso del Euc y Acah. El sitio SV con 38 días de quemado, fue el más afectado en sus propiedades químicas, posiblemente se debe a que al realizarse la quema sobre la superficie del suelo esta puede llegar a repercutir en toda la capa arable y la superficie, al quedar expuesta a la radiación solar post-quema y dado su color negro eleva la temperatura del mismo (Gleissman 2002). Los efectos en la perturbación de los suelos de acuerdo a lo encontrado en este pueden durar desde dos meses hasta 15 años dependiendo del tipo de manejo y del cultivo que se encuentre establecido. Este mismo 
patrón fue encontrado en las investigaciones de Lugo (1992) en plantaciones forestales de Puerto Rico, Zou (1993) en plantaciones forestales en Hawaii y Giardina et al. (2000) en selvas tropicales.

La única especie de lombriz encontrada fue Pontoscolex corethrurus de la familia Glososscolecidae, que pertenece a la categoría ecológica de las endógeas mesohúmicas, con origen exótico al ser originaria de la meseta de las Guyanas (Lavelle et al. 1987). Esta especie suele establecerse muy bien en suelos perturbados por los diferentes factores macro (precipitación, temperatura y radiación solar) y microambientales $(\mathrm{pH}$, materia orgánica, $\mathrm{N}, \mathrm{P}$, $\mathrm{S}$, entre otros), así como a las diversas técnicas de manejo de ambientes antropogénicos (Fragoso 2001, Lavelle \& Spain 2001, Edwards \& Bohlen 1996). Se le ha encontrado en 12 estados del país con 71 registros en diferentes ambientes, 5 naturales y 11 perturbados (Fragoso 2001). Estudios locales en Tabasco realizados por Huerta et al. (2007a) encontraron que $P$. corethrurus se estableció en los diferentes ambientes agrícolas. Ordaz-Chaparro et al. (1996) en suelos de la sabana de Huimanguillo, encontró que esta dominó sobre las otras especies en la mayoría de los sitios de selva, potreros y cultivos de cítricos con diferentes edades, asimismo, en Puerto Rico esta especie fue más abundante en cultivos forestales con respecto a sitios de Acahual donde había mayor diversidad de lombrices (González et al. 1996). Por último, Barros et al. (2003) en pastizales amazónicos abandonados llegaron a estar dominados por esta especie desplazando completamente a otras especies de lombrices.

Los estudios entorno a los oligoquetos ha demostrado que estos macroinvertebrados son excelentes indicadores de la calidad de los suelos (Velásquez et al. 2007, Huerta et al. 2009, Brown \& Domínguez 2010). Lo que podríamos señalar que el acahual a pesar de ser un sistema en regeneración por su abandono hace más de 15 años por la empresa FORMEX, sigue presentando rasgos de un sistema perturbado de vegetación secundaria y en vías de recuperación, como señalan Fragoso (2001) y Huerta et al. (2006) al estudiar lombrices en acahuales. Estos suelos han podido recuperar parte de su fauna de oligoquetos nativos con el paso del tiempo ( $\geq$ de 15 años en abandono) posiblemente a la colindancia que tiene este sitio con los cultivos de eucalipto en producción puede estar generándole algún efecto de borde por el constante manejo (uso de fertilizantes y pesticidas) que reciben estos durante el año por parte de la empresa FORMEX, lo que hace que solo $P$. corethrurus sea la única especie tolerante al manejo que se realiza en estos cultivos de Eucalyptus grandis, como en el caso de los pastizales de Manaus, Brazil, en donde Lavelle et al. (1997) determinaron como P. corethrurus ocupo nichos abandonados por las especies nativas producto de la perturbación de los suelos.

El hecho de que él área tiene niveles muy bajos en su $\mathrm{pH}$ puede ser uno de los principales factores que afectan la diversidad de lombrices, debido a que los vermes son sensibles a condiciones extremas de $\mathrm{pH}$ en el suelo, al preferir establecerse en suelos con rangos entre 4.5 y 7.5 (Reynolds 1994, Edwards \& Bohlen 1996). Lavelle et al. (1994), Huerta et al. (2006) mencionan que en sistemas tropicales las lombrices endógeas son más abundantes en suelos con pH 5-6. En nuestro país, las comunidades de estos vermes son dominadas por los endógeos (Fragoso 2001), por lo que el efecto del pH podría reflejarse más en la diversidad que en la estructura funcional. Algunos investigadores sugieren que los oligoquetos prefieren vivir agregadas en donde las condiciones de textura, materia orgánica, humedad y nutrientes son propicias para su desarrollo (Lee 1985, Edwards \& Bohlen 1996).

A pesar del paso del tiempo y la falta de manejo o reposo del sitio de Acahual este no recuperó especies de lombrices. Por lo tanto, se puede considerar que la diversidad de lombrices una vez alterada difícilmente se puede recuperar, similar a lo reportado en plantaciones de eucalipto en Paraná, Brasil (Dionisio et al. 1994) y Sudáfrica (Nadel et al. 2007).

En conclusión, a pesar de que el uso del fuego es parte de las técnicas utilizadas para 
eliminar de una manera más fácil los residuos vegetales después de la tala de árboles de eucalipto, en este estudio observamos como los tratamientos Acah, Euc, y SV variaron sus concentraciones en el conjunto de nutrientes del suelo con respecto al paso del tiempo, asimismo los sitios de reciente quema (SV) pierden biomasa y densidad de lombrices, mientras que la diversidad de oligoquetos es afectada con el establecimiento y manejo de las plantaciones de E. grandis.

\section{AGRADECIMIENTOS}

Los autores agradecemos el financiamiento a Fondos Mixtos Gobierno del Estado de Tabasco-CONACYT (TAB-2007-C09-74864) para realizar este estudio, dentro del proyecto: "Incremento de la fertilidad del suelo mediante el uso de lombrices de tierra, Tabasco". A la empresa Forestaciones Operativas Mexicanas (FOMEX) por facilitarnos el acceso a sus instalaciones y sistemas forestales. Al M.C. Samuel Cabrera, a los ingenieros Yoana Silva, Jorge Arcos del ITVH y demás estudiantes por el apoyo en campo.

\section{RESUMEN}

La presencia de oligoquetos en los ecosistemas puede indicar fertilidad del suelo, ya que estos organismos transportan, mezclan y entierran los residuos vegetales de la superficie al interior del suelo. Se caracterizó la comunidad de oligoquetos bajo sitios con diferentes periodos de establecimiento y manejo de plantaciones de Eucalyptus grandis, sin vegetación (SV), con cinco años en producción (Euc) y vegetación secundaria con 15 años (Acah) que han pasado por el proceso de tumba y quema en suelos de Acrisol en Huimanguillo, Tabasco; y se analizaron las propiedades físico-químicas del suelo (D.A., humedad, textura, $\mathrm{pH}$, Ntot, MO, P, K, CIC). La recolecta de lombrices se realizó al finalizar las lluvias (agosto-octubre 2007). Se muestreó en tres parcelas con seis réplicas en cada una. Se encontró que los suelos tenían $\mathrm{pH}$ de $3.0-4.5$ en los primeros $30 \mathrm{~cm}$ de profundidad. Los contenidos de materia orgánica (MO) y nitrógeno total (Ntot) fueron significativamente menores en los sitios SV (6-8\% y $0.19-0.22 \%$ respectivamente) que en Euc y Acah (MO=9-11\%; el Ntot=0.27-0.33\%). La especie Pontoscolex corethrurus domino en toda el área, presentando mayores densidades y biomasas en Euc $\left(164.4 \mathrm{ind} / \mathrm{m}^{2}\right.$ y $36.8 \mathrm{~g} / \mathrm{m}^{2}$ respectivamente) y Acah (138.7ind $/ \mathrm{m}^{2}$ y $19.1 \mathrm{~g} / \mathrm{m}^{2}$ respectivamente), mientras que en $\mathrm{SV}$ sus poblaciones fueron reducidas en un $80 \%$. Se encontró que el sistema Acah sigue presentando rasgos de un sistema perturbado, al no recuperar fácilmente la diversidad de oligoquetos y las concentraciones de nutrientes disponibles en el suelo.

Palabras claves: Pontoscolex corethrurus, lombrices de tierra, tumba y quema, Eucalyptus grandis, manejo, fertilidad del suelo.

\section{REFERENCIAS}

Aguilera, H.N. 1989. Tratado de edafología de México. Facultad de Ciencias, UNAM. México.

Almendros, G., F.J. González-Villa \& F. Martín. 1990. Fire-induced transformation of soil organic matter from an oak forest: an experimental approach to the effects of fire on humic substances. Soil. Sci. 149: 158-168.

Anderson, R.V. \& J.S. Ingram. 1993. Tropical Soil Biology and Fertility: A handbook of Methods. CAB International, Wallinford, United Kingdom.

Barros, E., A. Neves, E. Blanchart, E.C.M. Fernandes, E. Wandelli \& P. Lavelle. 2003. Development of the soil macrofauna community under silvopastoral and agrosilvicultural systems in Amazonia. Pedobiologia 47: 273-280.

Bigham, J.M. \& J.M. Bartels (eds.). 1996. Methods of soils analysis. Part 3, Soil Science Society of America Book Series. Chemical. Methods. American Society of Agronomy, Wisconsin, USA.

Bouché, M.B. 1984. Los Gusanos de Tierra. Mundo Científico 40: 954-963.

Bouyoucos, G. 1962. Hidrometer method for particle-size analysis of soils. Agr. J. 54: 464-465.

Brown, G.G. \& J. Domínguez. 2010. Uso das minhocas como bioindicadoras ambientais: princípios e práticas-O $3^{\circ}$ encontro latino americano de ecologia e taxonomia de oligoquetas (Elaetao 3). Acta Zool. Mex. 2: 1-18.

Brown, G., B. Pashanasi, C. Villenave, J.C. Patrón, B.K. Senapati, S. Giri, I. Barois, P. Lavelle, E. Blanchart, R.J. Blakemore, A.V. Spain \& J. Boyer. 1999. Effects of earthworms on plant production in the tropics, p. 87-147. In P. Lavelle, L. Brussaard \& P.F. Hendrix (eds.). Earthworms management in tropical agroecosystems. CAB International, Wallingford, United Kingdom.

Ceccon, E. \& M. Martinez-Ramos. 1999. Aspectos ambientales referentes al establecimiento de plantaciones de eucalipto de gran escala en áreas tropicales: aplicación al caso México. Interciencia 24: 352-359.

Certini, G. 2005. Effects of fire on properties of forest soils: a review. Oecologia 43: 1-10. 
Dionisio, J.A., B.C.B. Tanck, A. dos Santos, V.Y. Silveira, H.R. Santos \& H.R. dos Santos. 1994. Avaliação da população de Oligochaeta (terrestres) em áreas degradadas. Sci. Agrar. 13: 35-40.

Edwards, C.A. \& P.J. Bohlen. 1996. Biology and Ecology of Earthworms. Chapman \& Hall, London, United Kingdom.

FAO. 2009. La FAO en México. Más de 60 años de cooperación 1945-2009. Agroanálisis AC., D.F. México.

Fernández, C., J.A. Vega, J.M. Gras, T. Fonturbel, P. Cuiñas, E. Dambrine \& M. Alonso. 2004. Soil erosion after Eucalyptus globulus clearcutting: differences between logging slash disposal treatments. J. Forest. Ecol. Manag. 195: 85-95.

Fragoso, C. 1993. Les peuplements de vers de terre dans l'est et sud'est du Mexique. Thèse de doctorat, Université Paris 6, Paris, France.

Fragoso, C. 2001. Las lombrices de tierra de México (Annelida, Oligochaeta): Diversidad, Ecología y Manejo. Rev. Acta Zool. Mex. 1: 131-171.

Giardina, C.P., R.L. Sandford, I.C. Døckersmith \& V.J. Jaramillo. 2000. The effects of slash burning on ecosystem nutrients during the land preparation phase of shifting cultivation. Plant \& Soil 220: 247-260.

Gibbons, P., D.B. Lindermayer, S.C. Barry \& M.T. Tanton. 2000. The effects of slash burning on the mortality and collapse of trees retained on logged sites in southeastern Australia. J. Forest Ecol. Manag. 139: 51-61.

Gimeno-García, E., V. Andreu \& J.L. Rubio. 2000. Changes in organic matter, nitrogen and phosporus and cations as a result of fire and water erosion in a mediterranean landscape. Eur. J. Soil Sci. 51: 201-210.

Gliessman, S.R. 2002. Agroecología: procesos ecológicos en agricultura sostenible. CATIE, Turrialba, Costa Rica.

González, G., X. Zou \& S. Borges. 1996. Earthworm abundance and species composition in abandoned tropical croplands: comparisions of tree plantations and secondary forests. Pedobiologia 40: 385-391.

Gómez-Pompa, A. 1971. Posible papel de la vegetación secundaria en la evolución de la flora tropical. Biotropica 3: 125-135.

Huerta, E., C. Fragoso, J. Rodríguez-Olan, I. Evia-Castillo, E. Montejo-Meneses, M. De la Cruz-Mondragon \& R. García-Hernández. 2006. Presence of exotic and native earthworms in principal agro- and natural systems in Central and Southeastern Tabasco, Mexico. Caribb. J. Sci. 42: 359-365.

Huerta, E., J. Rodriguez-Olan, I. Evia-Castillo, E. MontejoMeneses, M. De la Cruz-Mondragón, R. GarcíaHernández \& S. Uribe. 2007a. Earthworms and soil properties in Tabasco, Mexico. Eur. J. Soil Biol. 43: 190-195.

Huerta, E., D. De la O \& G. Nuncio. 2007b. Incremento de la fertilidad del suelo mediante el uso de lombrices de tierra (Glossoscolecidae y Acanthodrilidae) y leguminosas (Arachys pintoi) en el suelo de traspatio. Rev. Ciencia Ergo Sum. 14: 172-176.

Huerta, E., C. Kampichler, V. Geissen, S. Ochoa-Gaona, B. de Jong \& S. Hernández-Daumás. 2009. Towards an ecological index for tropical soil quality based on soil macrofauna. Pesq. Agropec. Bras. 44: 1056-1062.

INEGI. 2001. Síntesis Geográfica, Nomenclátor y Anexo Cartográfico del Estado de Tabasco. Instituto nacional de Estadística, Geografía e Informática. Tabasco, México.

INEGI. 2005. Cuaderno estadístico del municipio de Huimanguillo, Tabasco. Instituto Nacional de Estadística, Geografía e Informática. Tabasco, México.

Jaramillo, V.J., J.K. Boone, L. Rentería-Rodríguez, D.L. Cummings \& L.J. Ellingson. 2003. Biomass, Carbon, and Nitrogen Pools in Mexican Tropical Dry Forest Landscapes. Ecosystems 6: 609-629.

Jouquet, P., J. Dauber, J. Lagerlöf, P. Lavelle \& M. Lepage. 2006. Soil invertebrates as ecosystem engineers: Intended and accidental effects on soil and feedback loop. App. Soil Ecol. 32: 153-164.

Kanowski, P.J. \& P.S. Savill. 1990. Plantation Forestry. World Bank Forest Policy Issues Paper. Oxford Forestry Institute, Oxford, United Kingdom.

Knoepp, J.D., J.M. Vose \& W.T. Swank. 2004. Long-term soil responses to site preparation burning in the southern appalachians. Forest Sci. 50: 540-550.

Korb, J.E., N.C. Johnson \& W.W. Covington. 2004. Slash pile burning effects on soil biotic and chemical properties and plant establishment: recommendations for ameloration. J. Ecol. Rest. 12: 55-62.

Lal, R. 1987. Tropical ecology and physical edaphology. Wiley, Nueva York, EEUU.

Lavelle, P. \& V. Spain. 2001. Soil Ecology. Kluwer Academic, Dordrecht, Holanda.

Lavelle, P., D. Bignell, M. Lepage, V. Woters, P. Roger, P. Ineson, O.W. Heal \& S. Dhillion. 1997. Soil function in a changing world: the role of invertebrates ecosystems engineers. Eur. J. Soil Biol. 33: 159-193.

Lavelle, P., I. Barois, C. Cruz, A. Hernández, A. Pineda \& P. Rangel. 1987. Adaptative strategies of Pontoscolex corethrurus (Glossoscolecidæ, Oligochaeta), a peregrine geophagous earthworm of the humid tropics. Biol. Fert. Soils 5: 188-194.

Lavelle, P., M. Dangerfield, C. Fragoso, V. Eschenbrenner, D. López-Hernández, B. Pashanasi \& L. Brussaard. 1994. The relationship between soil macrofauna and tropical soil fertility. p. 137-169. In P.L. Woomer \& M.J. Swift (eds.). The biological management of tropical soil fertility. Wiley, Nueva York, EEUU.

Lee, K.E. 1985. Earthworms. Their ecology and relationships with soils and land use. Academic, Sydney, Australia. 
Lugo, A.E. 1992. Comparison of tropical tree plantations with secondary forest of similar age, Ecol. Monogr. 62: $1-41$

Marín, A.A. 2006. Caracterización, clasificación y cartografía de suelos cítricos en Tabasco. Tesis de Maestría, Colegio de Postgraduados, Campus Tabasco, México.

Martínez-Ruiz, R., H.S. Azpiros-Rivero, J.L. RodríguezDe la O, V.M. Cetina-Alcalá \& M.A. GutierrézEspinosa. 2006. Importancia de las plantaciones forestales de Eucalyptus. Ra. Ximhai. Soc. Cult. Des. Sust. 2: 815-846.

Mengel, K. 1996. Turnover of organic nitrogen in soils and its availability to crops. Plant Soil 181: 83-93.

Nadel, R.L., M.C. Scholes \& M.J. Byrne. 2007. Slash burning, faunal composition, and nutrient dynamics in a Eucalyptus grandis plantation in South Africa. Can. J. For. Res. 37: 226-235.

Ochoa-Gaona, S., F. Hernández-Vázquez, B.H.J. De Jong \& F.D. Gurrí-García. 2007. Pérdida de diversidad florística ante un gradiente de intensificación del sistema agrícola de Roza-Tumba-Quema: un estudio de caso en la Selva Lacandona, Chiapas, México. Bol. Soc. Bot. Méx. 81: 65-80.

Olsen, S.R. \& L.A. Dean. 1965. Methods of soil analysis. Part 2. J. Agron. 9: 1035-1049.

Ordaz-Chaparro, M.V., I. Barois \& A. Aguilar-Santelises. 1996. Fauna del suelo de la Sabana de Huimanguillo alterada por cambios en el uso de la tierra. Terra 14: 387-393.

Palma-López, D.J. \& J.D. Cisneros. 2000. Plan de uso sustentable de los suelos de Tabasco. Fundación Produce Tabasco A.C. Villahermosa, Tabasco, México. In D.J. Palma-López, J.D. Cisneros, E.C. Moreno \& J.A. Rincón-Ramiréz. 2007. Suelos de Tabasco: su uso y manejo sustentable. Instituto del Trópico Húmedo, Colegio de Postgraduados y Fundación Produce Tabasco A.C. Tabasco, México.

Ponce-Mendoza, A., P. Boeckx, F. Gutierrez-Miceli, O. Van Cleemput \& L. Dendooven. 2006. Influence of water regime and $\mathrm{N}$ availability on the emission of nitrous oxide and carbon dioxide from tropical, semi-arid soils of Chiapas, Mexico. J. Arid. Envir. 64: 137-151.

Porta, C.J., R.M. López-Acevedo \& I.B.C. Roquero. 1999 Edafología para la Agricultura y el Medio Ambiente. Mundi Prensa, Madrid, España.

Rab, M.A. 1996. Soil physical and hydrological properties following logging and slash burning in the Eucalyptus regnans forest of southeastern Australia. Forest Ecol. Manag. 84: 159-176.

Reynolds, J. 1994. Earthworms of the world. Global Biodiv. 4: 11-16.

Ribeiro, C., M. Madeira \& M.C. Araújo. 2002. Decomposition and nutrient release from leaf litter of Eucalyptus globulus grown under different water and nutrient regimes. Forest Ecol. Manag. 171: 31-41

Román-Cuesta, R.M. 2000. Forest fire situation in the state of Chiapas, Mexico. In J. Pugliese. Global Forest Fire Assessment 1990-2000, working paper 55. Forestry department, FAO, Roma, Italia.

Satchell, J.E. 1967. Lumbricidae, p. 308-374. In A. Burges \& F. Raw (eds.). Soil Biology Academic, London.

SEMARNAT (Secretaría del Medio Ambiente y Recursos Naturales). 2000. Norma Oficial Mexicana NOM021-RECNAT-2000. SEMARNAT, México.

Sparks, D.L. 1996. Methods of soil analysis: Part 3. Chemical methods. ASA, SSSA, CSSA, Madison, Wisconsin, EEUU.

StatSoft. 2006. Statistica 06' Edition. StatSoft Inc.

Téllez-Mejía, E., M.J. González-Guillén, H.M. De los Santos-Posadas, A.M. Fierros-González, R. J. Lilieholm \& A. Gómez-Guerrero. 2008. Rotación óptima en plantaciones de eucalipto al incluir ingresos por captura de carbono en Oaxaca, México. Rev. Fitotecnia Mexicana 31: 173-182.

Turnbull, J.W. 1990. Eucalyptus plantations. New Forests 17: $37-52$.

Velásquez, E., P. Lavelle \& M. Andrade. 2007. GISQ a multifunctional indicator of soil quality. Soil Biol. Biochem. 39: 3066-3080.

Villar, M.C., V. Petrikova, M. Diáz-Raviña \& T. Carballas. 2004. Changes in soil microbial biomass and aggregate stability following burning and soil rehabilitation. Geoderma 122: 73-82.

Walkley, A. \& I.A. Black. 1934. An examination of degtjareff method for determining soil organic matter and a proposed modification of the chromic acid titration method. Soil Sci. 37: 29-37.

Willard, H.H., L.L. Merrit Jr. \& J.A. Dean. 1974. Instrumental methods of analysis. Van Nostrand, Nueva York, EEUU

Wilson, C.A., R.L. Mitchell, L.R. Boring \& J.J. Hendricks. 2002. Soil nitrogen Dynamics in a fire maintained forest ecosystem: results over a 3 year burn interval. Soil Biol. Biochem. 34: 679-689.

Wojtkowski, P.A. 2002. Agroecological perspectives in agronomy forestry and agroforestry. Science Publishers, Endfield, New Hampshire, EEUU.

Yang, Y.S., J. Guo, G. Chen, J. Xie, R. Gao, Z. Li \& J. Jin. 2005. Carbon and nitrogen pools in Chinese fir and evergreen broadleafed forests and changes associated with felling and burning in mid-subtropical China. J. For. Ecol. Manag. 216: 216-226.

Zou, X. 1993. Species effects on earthworm density in tropical tree plantations in Hawaii. Biol. Fert. Soils 15: 35-38. 